\title{
Consistency of Global Checkpoints Based on Characteristics of Communication Events in Multimedia Applications
}

\author{
Masakazu Ono and Hiroaki Higaki \\ Department of Computers and Systems Engineering, Tokyo Denki University \\ Hatoyama-cho Ishizaka, Hiki, Saitama, 350-0394, Japan \\ \{masa, hig\}@higlab.k.dendai.ac.jp
}

\begin{abstract}
In order achieving fault-tolerant network systems, checkpoint-recovery has been researched and many protocols have been designed. A global checkpoint taken by the protocols have to be consistent. For conventionalnetworks, a global checkpoint is defined to be consistent if there is no inconsistent message in any communication channel [1. For multimedia communication networks, there are additional requirements for time-constrained failure-free execution and large-size message transmissions where lost of a part of the message is acceptable. In addition, based on not characteristics of data of a message but characteristics of communication events for the message, restrictions of a consistent global checkpoint are determined. This reflects deterministic and non-deterministic property of the usage of communication buffers in layered system model. This paper proposes novel criteria, for global checkpoints in multimedia communication networks.
\end{abstract}

\section{Multimedia Networks}

In a multimedia network, it takes longer time to transmit and receive a message. Here, the following four pseudo events are defined for a multimedia message $\bar{m}$ transmitted through a communication channel $\left\langle p_{i}, p_{j}\right\rangle ; s b(\bar{m}), s e(\bar{m}), r b(\bar{m})$ and $r e(\bar{m})$ for start and end of sending $\bar{m}$ in $p_{i}$ and start and end of receiving $\bar{m}$ in $p_{j}$, respectively. In addition, $\bar{m}$ is decomposed into a sequence $\left\langle p a_{1}, \ldots, p a_{l}\right\rangle$ of multiple packets for transmission. Here, $s\left(p a_{k}\right)$ is a packet sending event and $r\left(p a_{k}\right)$ is a packet receipt event for a packet $p a_{k}$. A communication event in a multimedia network is characterized by when data of a transmitted packet is determined and when data of a received packet is accepted by an application.

Packet sending events are classified into bulky and stream ones. Data of a packet sent at a bulky packet sending event is determined at beginning of sending event of a multimedia message. Here, the following properties are held:

- If $s(p a)$ for $\bar{m}$ is bulky, $e \rightarrow s(p a)$ iff $e \Rightarrow s b(\bar{m})$ where $\rightarrow$ and $\Rightarrow$ represent causal precedence and temporal precedence between two events, respectively.

- If $s(p a)$ for $\exists p a \in \bar{m}$ is bulky, $s\left(p a^{\prime}\right)$ for $\forall p a^{\prime} \in \bar{m}$ is bulky. 
On the other hand, data of a packet $p a$ sent at a stream packet sending event is determined just at this event. Here, the following properties are held:

- If $s(p a)$ for $\bar{m}$ is stream, $e \rightarrow s(p a)$ iff $e \Rightarrow s(p a)$.

- If $s(p a)$ for $\exists p a \in \bar{m}$ is stream, $s\left(p a^{\prime}\right)$ for $\forall p a^{\prime} \in \bar{m}$ is stream.

Same as a packet sending event, packet receipt events, are also classified into bulky and stream ones. Data of a packet received at a bulky packet receipt event is accepted at ending of receipt event of a multimedia message. Here, the following properties are held:

- If $r(p a)$ for $\bar{m}$ is bulky, $r(p a) \rightarrow e$ iff $r e(\bar{m}) \Rightarrow e$.

- If $r(p a)$ for $\exists p a \in \bar{m}$ is bulky, $r\left(p a^{\prime}\right)$ for $\forall p a^{\prime} \in \bar{m}$ is bulky.

On the other hand, data of a packet received at a stream packet sending event is accepted just at this event. Here, the following properties are held:

- If $r(p a)$ for $\bar{m}$ is stream, $r(p a) \rightarrow e$ iff $r(p a) \Rightarrow e$.

- If $r(p a)$ for $\exists p a \in \bar{m}$ is stream, $r\left(p a^{\prime}\right)$ for $\forall p a^{\prime} \in \bar{m}$ is stream.

For a message $m$ with conventional data, $s(m)$ and $r(m)$ are atomic. Here, each $c_{i}$ in $p_{i}$ is taken only when no other event occurs in $p_{i}$. However, a multimedia message is so larger that it takes longer time to transmit and receive the message. Thus, if $p_{i}$ is required to take $c_{i}$ during a communication event, it has to wait until an end of the event. Hence, timeliness requirement in a checkpoint protocol is not satisfied and communication overhead in recovery is increased. Therefore, $c_{i}$ should be taken immediately when $p_{i}$ is required to take it even during a communication event. That is, $p_{i}$ sending $\bar{m}=\left\langle p a_{1}, \ldots, p a_{l}\right\rangle$ takes $c_{i}$ between $s\left(p a_{s}\right)$ and $s\left(p a_{s+1}\right)$ and $p_{j}$ receiving $\bar{m}$ takes $c_{j}$ between $r\left(p a_{r}\right)$ and $r\left(p a_{r+1}\right)$. In addition, part of a multimedia message may be lost in a communication channel for an application. Such an application requires not to retransmit lost packets in recovery but to transmit packets with shorter transmission delay. Hence, an overhead for taking a checkpoint during failure-free execution is required to be reduced.

\section{Consistency in Multimedia Networks}

Global consistency $G c$ denotes degree of consistency for a global checkpoint $C_{\mathcal{V}}=\left\{c_{1}, \ldots, c_{n}\right\}$. In a conventional network, $G c$ is defined as follows:

$$
G c= \begin{cases}1 & \text { no inconsistent message } \\ 0 & \text { otherwise }\end{cases}
$$

In a multimedia network, a local checkpoint is taken even during a communication event and it is acceptable to lose part of a multimedia message. Hence, a domain of $G c$ is a closed interval $[0,1]$ instead of a discrete set $\{0,1\}$.

\subsection{Message Consistency}

Message consistency. $M c_{i j}^{u}$ is degree of consistency for a set $\left\{c_{i}, c_{j}\right\}$ of local checkpoints and a multimedia message $\overline{m_{u}}$ through a communication channel $\left\langle p_{i}, p_{j}\right\rangle$. Here, we define an inconsistent multimedia message. 
[Inconsistent multimedia message.] $m_{u}$ is inconsistent iff $m_{u}$ is a lost or an orphan multimedia message. $m_{u}$ is a lost multimedia message iff $s e\left(\overline{m_{u}}\right)$ occurs before $c_{i}$ in $p_{i}$ and $r b\left(\overline{m_{u}}\right)$ occurs after $c_{j}$ in $p_{j} . m_{u}$ is an orphan multimedia message iff $s b\left(\overline{m_{u}}\right)$ occurs after $c_{i}$ in $p_{i}$ and $r b\left(\overline{m_{u}}\right)$ occurs before $c_{j}$ in $p_{j}$.

[Consistency for inconsistent multimedia message.] $M c_{i j}^{u}=0$ for an inconsistent multimedia message $\overline{m_{u}}$.

Next, inconsistent packets are introduced due to checkpoints during a communication event.

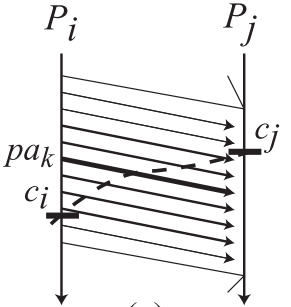

(a)

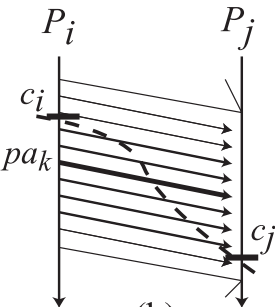

(b)

Fig. 1. Lost packet (a) and Orphan packet (b).

[Lost and orphan packets.] Suppose that $c_{i}$ and $c_{j}$ are between $s\left(p a_{s}\right)$ and $s\left(p a_{s+1}\right)$ and between $r\left(p a_{r}\right)$ and $r\left(p a_{r+1}\right)$ for $\overline{m_{u}}=\left\langle p a_{1}, \ldots, p a_{l}\right\rangle$, respectively. $p a_{k}$ is a lost packet iff $s\left(p a_{k}\right)$ occurs before $c_{i}$ in $p_{i}$ and $r\left(p a_{k}\right)$ occurs after $c_{j}$ in $p_{j} \cdot p a_{k}$ is an orphan packet iff $s\left(p a_{k}\right)$ occurs after $c_{i}$ in $p_{i}$ and $r\left(p a_{k}\right)$ occurs before $c_{j}$ in $p_{j}$. $\square$

If $s=r$, there is no lost and orphan packet. Hence, $M c_{i j}^{u}=1$.

If $s>r,\left\{p a_{r+1}, \ldots, p a_{s}\right\}$ is a set of lost packets. These packets are not retransmitted after recovery. Lost packets in a conventional network are restored by logging them in failure-free execution. However, in a multimedia network, less overhead in failure-free execution is required since applications require timeconstrained execution. In addition, even if part of a multimedia message is lost in recovery, an application accepts the message. The less packets are lost, the higher message consistency is achieved. A multimedia message is usually compressed for transmission. Thus, value of packets for a message is not unique. Therefore, message consistency depends on total value of lost packets as follows:

$$
\frac{\partial M c_{i j}^{u}}{\partial l \text {-value }}<0 \text { where l-value }=\sum_{\text {lost packets pa }} \operatorname{value}\left(p a_{k}\right) .
$$

Here, a domain of $M c_{i j}^{u}$ is an open interval $(0,1)$.

If $s<r,\left\{p a_{s+1}, \ldots, p a_{r}\right\}$ is a set of orphan packets. An orphan multimedia message might not be retransmitted after recovery. However, orphan packets are surely retransmitted since $c_{i}$ and $c_{j}$ are taken during transmission and receipt of $\overline{m_{u}}$ and the data of $\overline{m_{u}}$ being carried by a sequence $\left\langle p a_{1}, \ldots, p a_{l}\right\rangle$ of packets is not changed even after recovery. Hence, message consistency does not depend on orphan packets. 
[Message consistency] Let value $\left(\overline{m_{u}}\right)$ be total value of all packets in $\overline{m_{u}}$.

$$
\begin{aligned}
M c_{i j}^{u}=0 & \text { if l-value }=\operatorname{value}\left(\overline{m_{u}}\right) . \\
M c_{i j}^{u}=1 & \text { if l-value }=0 . \\
\frac{\partial c_{i j}^{u}}{\partial l \text {-value }}<0 & \text { otherwise. }
\end{aligned}
$$

\subsection{Channel Consistency}

Channel Consistency. $C c_{i j}$ is calculated by $M c_{i j}^{u}$ for every message $\overline{m_{u}}$ through $\left\langle p_{i}, p_{j}\right\rangle$. For compatibility with (1), if message consistency for every message through $\left\langle p_{i}, p_{j}\right\rangle$ is 1 , channel consistency is also 1 . On the other hand, if message consistency for at least one message through $\left\langle p_{i}, p_{j}\right\rangle$ is 0 , channel consistency is also 0 . In addition, channel consistency monotonically increases for consistency of messages through $\left\langle p_{i}, p_{j}\right\rangle$.

[Channel consistency.] Let $\mathcal{M}_{i j}$ be a set of messages through $\left\langle p_{i}, p_{j}\right\rangle$.

$$
\begin{array}{rlrl}
C c_{i j} & =1 & & \text { if } \forall \overline{m_{u}} \in \mathcal{M}_{i j} \quad M c_{i j}^{u}=1 . \\
C c_{i j}=0 & & \text { if } \exists \overline{m_{u}} \in \mathcal{M}_{i j} \quad M c_{i j}^{u}=0 . \\
\forall \overline{m_{u}} \in \mathcal{M}_{i j}, \frac{\partial C c_{i j}}{\partial M c_{i j}^{u}}>0 & & \text { otherwise. }
\end{array}
$$

\subsection{Global Consistency}

$G c$ is calculated by $C c_{i j}$ for every communication channel $\left\langle p_{i}, p_{j}\right\rangle$. For compatibility with (1), if channel consistency for every channel is 1 , global consistency is also 1 . On the other hand, if consistency for at least one communication channel is 0 , global consistency is also 0 . In addition, channel consistency monotonically increases for consistency of the communication channels.

[Global consistency.]

$$
\begin{array}{rlrl}
G c=1 & & \text { if } \forall\left\langle p_{i}, p_{j}\right\rangle \quad C c_{i j}=1 . \\
G c=0 & \text { if } \exists\left\langle p_{i}, p_{j}\right\rangle \quad C c_{i j}=0 . \\
\forall\left\langle p_{i}, p_{j}\right\rangle, \frac{\partial G c}{\partial C c_{i j}}>0 & & \text { otherwise. }
\end{array}
$$

\section{Conclusion}

This paper has proposed a novel criteria for consistency of a global checkpoint in multimedia network systems. The authors will design QoS-based checkpoint protocols based on the criteria.

\section{References}

1. Chandy, K.M. and Lamport, L., "Distributed Snapshots: Determining Global States of Distributed Systems," ACM Trans. on Computer Systems, Vol. 3, No. 1, pp. 63-75 (1985). 